\title{
An Implicit Two-Point Numerical Integration Formula for Linear and Nonlinear Stiff Systems of Ordinary Differential Equations
}

\author{
By Simeon Ola. Fatunla
}

\begin{abstract}
In [1], the author proposed a semi-implicit one-step integration formula which effectively copes with linear systems of ordinary differential equations with widely varying eigenvalues. The integration algorithm is based on a local representation of the theoretical solution to the initial value problem by a linear combination of exponential functions. The resultant integration formula is of order four. Unfortunately, this algorithm cannot cope with nonlinear stiff systems of ordinary differential equations. In this paper, the author extends the concept adopted in [1] to construct an implicit two-point formula which can effectively cope with nonlinear stiff systems. The resultant integration formula is of order five and it is $L$-stable and convergent.
\end{abstract}

1. Introduction. We shall consider initial value problems of ordinary differential equations of the form

$$
\mathrm{y}^{\prime}=\mathrm{f}(x, \mathrm{y}), \quad \mathrm{y}(a)=\eta,
$$

where $y=\left({ }^{1} y,{ }^{2} y, \ldots,{ }^{m} y\right)^{T}$ and $\eta=\left({ }^{1} \eta, \ldots,{ }^{m} \eta\right)^{T}$. It is assumed that the function $\mathrm{f}(x, y)$ is defined and continuous in the region $\Gamma$ defined as

$$
\Gamma=R \times R_{m}, \text { where } R=a \leqslant x \leqslant b,
$$

is a finite interval on the real line and $y \in R_{m}$ implies $\|y\|_{\infty}<\infty$. In addition, the function $\mathrm{f}(x, y)$ also satisfies a Lipschitz condition of order one with respect to $y$.

Definition 1. The nonlinear initial value problem (1.1) is said to be stiff over the interval $R$ if for every $x \in R$, the eigenvalues $\left(\lambda_{s}(x), s=1, \ldots, m\right)$ of the Jacobian $(\partial \mathrm{f} / \partial \mathrm{y})$ satisfy the following conditions

$$
\begin{aligned}
& \text { (a) } \operatorname{Re} \lambda_{s}(x)<0, \quad s=1, \ldots, m, \\
& \text { (b) } \max \left|\frac{\operatorname{Re} \lambda_{r}(x)}{\operatorname{Re} \lambda_{s}(x)}\right| \gg 1, \quad r, s=1, \ldots, m .
\end{aligned}
$$

Let

$$
x_{t}=a+t h, \quad t=0,1, \ldots, n,
$$

represent a uniform subdivision of the interval of integration $R$ with the steplength $h$ given by

Received July 15, 1976; revised February 22, 1977.

AMS (MOS) subject classifications (1970). Primary 65L05; Secondary 65D30.

Key words and phrases. Stiff systems, ordinary differential equations, stiffness parameters, $A$-stable, $L$-stable, interpolating functions, eigenvalues, stepsize, Lipschitz constants. 


$$
h=(b-a) / n
$$

for a fixed integer $n$.

As in [1], we approximate the theoretical solution to problem (1.1) over every subinterval $I_{t}=x_{t} \leqslant x \leqslant x_{t+1}$ by the interpolating function

$$
\mathbf{F}(x)=\left(I-e^{\Lambda_{1} x}\right) \mathbf{A}-\left(I-e^{-\Lambda_{2} x}\right) \mathbf{B}+\mathbf{C},
$$

where $\mathbf{A}, \mathbf{B}$ and $\mathbf{C}$ are $m$-vectors with real entries ${ }^{i} A,{ }^{i} B,{ }^{i} C$ and $I$ an $m \times m$ identity matrix whilst $\Lambda_{1}$ and $\Lambda_{2}$ are $m \times m$ diagonal matrices with complex entries given by

$$
{ }^{i} \alpha_{1},{ }^{i} \alpha_{2}, \quad i=1,2, \ldots, m, \text { respectively. }
$$

2. Derivation of the Integration Formula. If $y_{t}$ denotes the numerical approximation to the theoretical solution $\mathrm{y}\left(x_{t}\right)$ at $x=x_{t}$ and $\mathrm{f}_{t}=\mathrm{f}\left(x_{t}, \mathrm{y}_{t}\right)$, we impose the following constraints on the interpolating function (1.6):

(a) that the interpolating function coincides with the theoretical solution at the endpoints of the subinterval $I_{t}$ : This implies that

$$
\mathbf{y}_{t+j}=\mathbf{F}\left(x_{t+j}\right), \quad j=0,1 ;
$$

(b) that the first derivative of the interpolating function coincides with the righthand side of $(1.1)$ at the endpoints of subinterval $I_{t}$. This also implies that

$$
\mathbf{f}_{t+j}=F^{\prime}\left(x_{t+j}\right), \quad j=0,1 .
$$

With these two sets of constraints, we readily obtain the following integration formula

$$
y_{t+1}=\mathbf{y}_{t}+(\gamma+\sigma) \mathbf{f}_{t}+\theta \mathbf{f}_{t+1},
$$

where $\gamma, \sigma$, and $\theta$ are $m$ by $m$ diagonal matrices with entries given by

$$
\begin{aligned}
& i_{\gamma}=\frac{\left(1-e^{i_{\alpha_{1} h}}\right) e^{-i_{\alpha_{2} h}}}{i_{\alpha_{1}}\left(e^{-i_{\alpha_{2}} h}-e^{i_{\alpha_{1} h}}\right)} \\
& i_{\sigma}=\frac{\left(1-e^{-i_{\alpha_{2} h}}\right) e^{i_{\alpha_{1} h}}}{i_{\alpha_{2}}\left(e^{-i_{\alpha_{2} h}}-e^{i_{\alpha_{1} h}}\right)},
\end{aligned}
$$

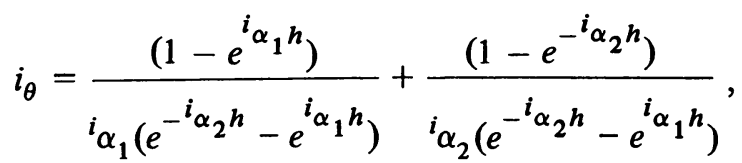

respectively, for $i=1,2, \ldots, m$.

The integration formula (2.3) can be expressed in the iterative form:

$$
\mathbf{y}_{t+1}^{[s+1]}=\mathbf{y}_{t}-(\gamma+\sigma) \mathbf{f}_{t}+\theta \mathbf{f}\left(x_{t+1}, \mathbf{y}_{t+1}^{[s]}\right) .
$$

We now have to obtain numerical estimates for the elements of $\Lambda_{1}$ and $\Lambda_{2}$ (i.e. the stiffness parameters). 
By using the Taylor series expansion of ${ }^{i} y_{t+1} \equiv y^{i}\left(x_{t+1}\right)$ about $x=x_{t}$, and the Maclaurin series expansion of $e^{i_{\alpha_{1}} h}$ and $e^{-i_{\alpha_{2}} h}$ in (2.3), we readily obtain the following identity:

$$
\begin{aligned}
\left(\sum_{r=1}^{\infty} \frac{h^{r} f_{t}^{(r-1)}}{r !}\right) & {\left[\sum_{r=0}^{\infty} \frac{\left(-{ }^{i} \alpha_{2} h\right)^{r}}{r !}-\sum_{r=0}^{\infty} \frac{\left({ }^{i} \alpha_{1} h\right)^{r}}{r !}\right] } \\
= & \left(-h \sum_{r=1}^{\infty} \frac{\left({ }^{i} \alpha_{1} h\right)^{r-1}}{r !}\right)\left[\sum_{r=0}^{\infty} \frac{h^{r}{ }^{i} f_{t}^{(r)}}{r !} \sum_{r=0}^{\infty} \frac{\left(-{ }^{i} \alpha_{2} h\right)^{r}{ }^{i} f_{t}}{r !}\right] \\
& -\left(h \sum_{r=1}^{\infty}(-1)^{r} \frac{\left({ }^{i} \alpha_{2} h\right)^{r-1}}{r !}\right)\left[\sum_{r=0}^{\infty} \frac{h^{r} i f_{t}^{(r)}}{r !} \sum_{r=0}^{\infty} \frac{\left({ }^{i} \alpha_{1} h\right)^{r} i f_{t}}{r !}\right],
\end{aligned}
$$

As the coefficients of $h^{0}, h^{1}$ and $h^{2}$ vanish identically in (2.8), we require that the coefficients of $h^{4}$ and $h^{5}$ vanish to give the following $m$ pairs of equations:

$$
\left({ }^{i} \alpha_{1}+{ }^{i} \alpha_{2}\right){ }^{i} f_{t}^{(2)}-\left({ }^{i} \alpha_{1}^{2}-{ }^{i} \alpha_{2}^{2}\right){ }^{i} f_{t}^{(1)}-{ }^{i} \alpha_{1}{ }^{i} \alpha_{2}\left({ }^{i} \alpha_{1}+{ }^{i} \alpha_{2}\right){ }^{i} f_{t} \equiv 0
$$

and

$$
\left({ }^{i} \alpha_{1}+{ }^{i} \alpha_{2}\right)^{i} f_{t}^{(3)}-\left({ }^{i} \alpha_{1}^{3}+{ }^{i} \alpha_{2}^{3}\right)^{i} \cdot f_{t}^{(1)}-{ }^{i} \alpha_{1}{ }^{i} \alpha_{2}\left({ }^{i} \alpha_{1}^{2}-{ }^{i} \alpha_{2}^{2}\right)^{i} f_{t} \equiv 0
$$

$$
\text { for } i=1,2, \ldots, m \text {. }
$$

Since we are not interested in the case of double eigenvalues, we divide both equations (2.9) and (2.10) by $\left({ }^{i} \alpha_{1}+{ }^{i} \alpha_{2}\right)$ to yield

$$
{ }^{i} f_{t}^{(2)}-\left({ }^{i} \alpha_{1}-{ }^{i} \alpha_{2}\right)^{i} f_{t}^{(1)}-{ }^{i} \alpha_{1}{ }^{i} \alpha_{2}{ }^{i} f_{t} \equiv 0
$$

and

$$
{ }^{i} f_{t}^{(3)}-\left({ }^{i} \alpha_{1}^{2}-{ }^{i} \alpha_{1}{ }^{i} \alpha_{2}+{ }^{i} \alpha_{2}^{2}\right)^{i} f_{t}^{(1)}-{ }^{i} \alpha_{1}{ }^{i} \alpha_{2}\left({ }^{i} \alpha_{1}-{ }^{i} \alpha_{2}\right){ }^{i} f_{t} \equiv 0
$$

Equations (2.11) and (2.12) can be readily transformed to the following pair of equations:

$$
\left({ }^{i} \alpha_{2}-{ }^{i} \alpha_{1}\right)^{i} f_{t}^{(1)}-{ }^{i} \alpha_{1}{ }^{i} \alpha_{2}{ }^{i} f_{t}=-{ }^{i} f_{t}^{(2)}
$$

and

$$
\left({ }^{i} \alpha_{2}-{ }^{i} \alpha_{1}\right)^{i} f_{t}^{(2)}-{ }^{i} \alpha_{1}{ }^{i} \alpha_{2}{ }^{i} f_{t}^{(1)}=-{ }^{i} f_{t}^{(3)}
$$

We now solve $m$ pairs of equations (2.13) and (2.14) (for ${ }^{i} \alpha_{1},{ }^{i} \alpha_{2}, i=1, \ldots, m$ ) using any of the Newton-like schemes. In all the numerical experiments of Section 6 , we shall adopt the Brown scheme as proposed in [2].

3. Problem with the Convergence of Formula (2.7). In order that the iteration formula (2.7) should converge, we do require that the relation 


$$
L\|\theta\|_{\infty}<1
$$

is satisfied where $L$ is the Lipschitz constant for $\mathrm{f}(x, \mathrm{y})$ with respect to $\mathrm{y}$. Apparently, this Lipschitz constant is very large for stiff systems and consequently, for condition (3.1) to be satisfied, the steplength $h$ must be extremely small.

With the view of making the integration formula (2.7) viable, we adopt the principle of the Newton method for solving nonlinear systems of equations of the form

$$
\mathbf{G}(y)=0 .
$$

The solution to (3.2) is given by

$$
\mathrm{y}^{[s+1]}=\mathrm{y}^{[s]}-J^{-1}\left(\mathrm{y}^{[s]}\right) \mathbf{G}\left(\mathrm{y}^{[s]}\right), \quad s=0,1, \ldots,
$$

where $J(\mathbf{y})$ is the Jacobian matrix $\delta \mathbf{G}(y) / \delta \mathbf{y}$.

This modification transforms the integration formula (2.7) into the form

$$
\begin{aligned}
\mathrm{y}_{t+1}^{[s+1]}= & \mathrm{y}_{t+1}^{[s]}-\left[I-\theta \frac{\delta \mathrm{f}^{2}}{\delta \mathrm{y}}\left(x_{t+1}, \mathrm{y}_{t+1}^{[s]}\right)\right]^{-1} \\
& \times\left[\mathrm{y}_{t+1}^{[s]}-\theta \mathrm{f}\left(x_{t+1}, \mathrm{y}_{t+1}^{[s]}\right)-\mathrm{y}_{t}+(\gamma+\sigma) \mathrm{f}_{t}\right], s=0,1, \ldots
\end{aligned}
$$

The final integration formula (3.4) when applied to stiff systems generally converges to the solution in at most three iterations without any restriction imposed on the steplength $h$. We normally use the numerical solution at $x=x_{t}$ as the starting value in the iteration (3.4); that is

$$
y_{t+1}^{[0]}=y_{t} .
$$

4. Stability. By applying the integration formula (2.3) to the scalar test equation

$$
y^{\prime}=\lambda y,
$$

where $\lambda$ is a complex constant with negative real part, we obtain

$$
\frac{y_{t+1}}{y_{t}}=\frac{P\left(\alpha_{1}, \alpha_{2}, \lambda, h\right)}{Q\left(\alpha_{1}, \alpha_{2}, \lambda, h\right)},
$$

where

and

$$
\begin{aligned}
P\left(\alpha_{1}, \alpha_{2}, \lambda, h\right)= & \alpha_{1} \alpha_{2}\left(e^{-\alpha_{2} h}-e^{\alpha_{1} h}\right)-\lambda \alpha_{2}\left(1-e^{\alpha_{1} h}\right) e^{-\alpha_{2} h} \\
& -\lambda \alpha_{1}\left(1-e^{-\alpha_{2} h}\right) e^{\alpha_{1} h},
\end{aligned}
$$

$$
\begin{aligned}
Q\left(\alpha_{1}, \alpha_{2}, \lambda, h\right)= & \alpha_{1} \alpha_{2}\left(e^{-\alpha_{2} h}-e^{\alpha_{1} h}\right) \\
& -\lambda \alpha_{2}\left(1-e^{\alpha_{1} h}\right)-\lambda \alpha_{1}\left(1-e^{-\alpha_{2} h}\right) .
\end{aligned}
$$

By setting $\alpha_{1}=\lambda$ or $-\alpha_{2}=\lambda$ in Eqs. (4.2) to (4.4), we have that

$$
y_{t+1} / y_{t}=e^{\lambda h},
$$

and $\left|e^{\lambda h}\right|<1$ as $\operatorname{Re} \lambda<0$. 
Hence, the numerical integration formulae (2.3), (2.7), (3.4) are $L$-stable and hence $A$-stable and also exponentially fitted.

5. Order and Local Truncation Error. We associate with the integration formula (2.3) and the constraints (2.13) and (2.14), the operator $P[y(x), h]$ which is defined as

$$
P[\mathrm{y}(x), h]=\mathrm{y}(x+h)-\mathrm{y}(x)+(\gamma+\sigma) \mathrm{f}(x, \mathrm{y})-\theta \mathrm{f}(x+h, \mathrm{y}(x+h)) .
$$

The local truncation error $T_{t+1}$ at $x=x_{t+1}$ is hence given by $P\left[y\left(x_{t}\right), h\right]$ with $y\left(x_{t}\right)$ assumed to be the theoretical solution to the problem (1.1). By assuming that $y_{t}=y\left(x_{t}\right)$, the truncation error $T_{t+1}$ for the integration formula (2.3) is readily obtained as:

$$
\begin{aligned}
& T_{t+1}=y\left(x_{t+1}\right)-y_{t+1} \\
& =\frac{h^{6}}{720}\left\{9 f_{t}^{(4)}+5\left(\alpha_{2}-\alpha_{1}\right) f_{t}^{(3)}+5\left(\alpha_{1}^{2}-\alpha_{1} \alpha_{2}+\alpha_{2}^{2}\right) f_{t}^{(2)}\right. \\
& -9\left(\alpha_{2}-\alpha_{1}\right)\left(\alpha_{1}^{2}+\alpha_{2}^{2}\right) f_{t}^{(1)} \\
& \left.+\left[5 \alpha_{1}^{2} \alpha_{2}^{2}-9 \alpha_{1} \alpha_{2}\left(\alpha_{1}^{2}-\alpha_{1} \alpha_{2}+\alpha_{2}^{2}\right)\right] f_{t}\right\}+O\left(h^{7}\right) .
\end{aligned}
$$

The last equation suggests that the numerical integration formula (2.3) with the constraints (2.13) and (2.14) is of order five.

6. Experimental Results. All the numerical experiments were implemented on an IBM 360/25 located at University of Benin (Nigeria). Numerical results are obtained for both linear and nonlinear stiff systems of ordinary differential equations using double precision arithmetic. For linear systems of ordinary differential equations of order $m$, the stiffness parameters have constant values in the interval of integration. Hence, the $m$ pairs of nonlinear equations (2.13) and (2.14) are only solved once for the stiffness parameters at the first step of the integration procedure. In all the numerical experiments, unit initial values are assigned to these stiffness parameters. Also, the iteration of the integration formula (3.4) is halted whenever the condition $\left\|y_{t+1}^{[s+1]}-y_{t+1}^{[s]}\right\|_{\infty}<10^{-8}$ is satisfied. Almost invariably, this condition is satisfied in less than three iterations in all the test runs. The stiffness parameters are obtained correctly to six decimal places in all the test runs.

The following initial value problems were considered in the interval $0 \leqslant x \leqslant 100$.

Problem 1.

\section{SYSTEM}

$$
\begin{aligned}
& y^{\prime}=-200(y-E(x))+E^{\prime}(x) \\
& E(x)=10-(10+x) e^{-x} \\
& y(0)=10
\end{aligned}
$$

\section{EXACT SOLUTION}

$y(x)=E(x)+10 e^{-200 x}$ 
Problem 2.

SYSTEM

$$
\begin{aligned}
& { }^{1} y^{\prime}=-2000^{1} y_{1}+1000^{2} y+1 \\
& { }^{2} y^{\prime}={ }^{1} y-{ }^{2} y \\
& { }^{1} y(0)={ }^{2} y(0)=0
\end{aligned}
$$

\section{EXACT SOLUTION}

$$
\begin{aligned}
& { }^{1} y(x)=-4.97{ }_{10^{-4}} e^{-2000.5 x} \\
& -5.034{ }_{10^{-4}} e^{-0.5 x}+0.001 \\
& { }^{2} y(x)=2.5{ }_{10^{-7} e^{-2000.5 x}} \\
& -1.007{ }_{10^{-3}} e^{-0.5 x}+0.001
\end{aligned}
$$

Problem 3.

SYSTEM

EXACT SOLUTION

${ }^{1} y^{\prime}=0.01-\left[1+\left({ }^{1} y+1000\right)\left({ }^{1} y+1\right)\right]\left[0.001+{ }^{1} y+{ }^{2} y\right]$

${ }^{2} y^{\prime}=0.01-\left(1+{ }^{2} y^{2}\right)\left(0.01+{ }^{1} y+{ }^{2} y\right)$

UNKNOWN

Problem 1 has been solved by Lapidus and Seinfeld [4] with the following numerical integration schemes:

\section{Scheme}

1. Fourth Order Runge Kutta

2. Fourth Order Predictor Corrector (Adams)

3. Treanor's Method

4. Trapezoidal Rule

5. Trapezoidal Rule with Extrapolation

6. Liniger and Willoughby (1)

7. Liniger and Willoughby (2)
Designation

RK4

DEQ

TM

TR

TR-EXTR

LW1

LW2

The same problem was also solved in Fatunla [1].

Problem 2 has also been solved in Lambert [3] and Fatunla [1] whilst Problem 3 has been solved in Lambert [3].

Example 1. The theoretical solution to this scalar initial value problem has a rapidly decaying component as well as a slowly decaying component thus posing the same stability problem as for stiff systems. With the integration formula (3.4), we generate the numerical solution to Problem 1 in the range $0 \leqslant x \leqslant 100$ using two different uniform mesh sizes $h=0.1$ and $h=0.2$. The numerical estimates of the stiffness parameters are:

$$
\alpha_{1}=-0.8890128 \text { and } \alpha_{2}=-200.000000 \text {. }
$$

More accurate results are obtained than in [1] as the new scheme is of higher order. Although, the relative error is slightly smaller at $x=0.4$ by using $h=0.1$, the relative errors are of the same magnitude at $x=10$ for both mesh sizes. This is perhaps due 
to the accumulation in the roundoff errors. The numerical and the theoretical solution coincide in the interval $42.8 \leqslant x \leqslant 100$.

Details of numerical results are given in Table 6.1 below:

TABLE 6.1

Relative Errors

$\begin{array}{llll}\text { Method } & \underline{h} & \underline{x}=0.4 & \frac{x=10.0}{1.0_{10^{-5}}} \\ \text { RK4 } & 0.01 & 2.0_{10^{-9}} \\ \text { DEQ } & 0.005 & 3.0_{10^{-9}} & 2.0_{10^{-9}} \\ \text { TM } & 0.2^{a} & 6.7_{10^{-8}} & 1.0_{10^{-9}} \\ \text { TR } & 0.2 & 1.85_{10^{-2}} & 4.3_{10^{-5}} \\ \text { TR-EXTR } & 0.2 & 1.4_{10^{-4}} & 1.0_{10^{-8}} \\ \text { LW1 } & 0.2 & 1.1_{10^{-3}} & 5.0_{10^{-8}} \\ \text { LW2 } & 0.2 & 1.8_{10^{-3}} & 9.0_{10^{-8}} \\ \text { FATUNLA [1] } & 0.2 & 2.9_{10^{-5}} & 1.8_{10^{-7}} \\ \text { FATUNLA [1] } & 0.1 & 1.4_{10^{-5}} & 1.1_{10^{-7}} \\ \text { FORMULA(3.4) } & 0.2 & 1.38_{10^{-6}} & 8.29 \\ \text { FORMULA(3.4) } & 0.1 & 1.36_{10^{-8}} & 8.42_{10^{-8}}\end{array}$

Index

$a$ automatic stepsize

maximum iterations of formula (3.4) is 2

Example 2. This problem is derived from Warten and Fowler [5] which is a linear system with constant coefficients and eigenvalues $\lambda_{1}=-2000.5$ and $\lambda_{2}=-0.5$. The numerical integration was implemented with a uniform mesh size $h=0.1$ in the interval $0 \leqslant x \leqslant 100$. The numerical estimates of the stiffness parameters are

$$
\Lambda_{1}=\left(\begin{array}{cc}
-0.49987499 & 0 \\
0 & -0.49987500
\end{array}\right), \quad \Lambda_{2}=\left(\begin{array}{cc}
2000.50012 & 0 \\
0 & 2000.50012
\end{array}\right)
$$

The details of the numerical experiments are given below in Table 6.2.

Example 3. This is the nonlinear initial value problem of reaction kinetics from Liniger and Willoughby [6]. The eigenvalues of the system at $x=0$ are $\lambda_{1}=-1012$, $\lambda_{2}=-0.01$ and at $x=100$ the eigenvalues are:

$$
\lambda_{1}=-21.7, \quad \lambda_{2}=-0.089 \text {. }
$$




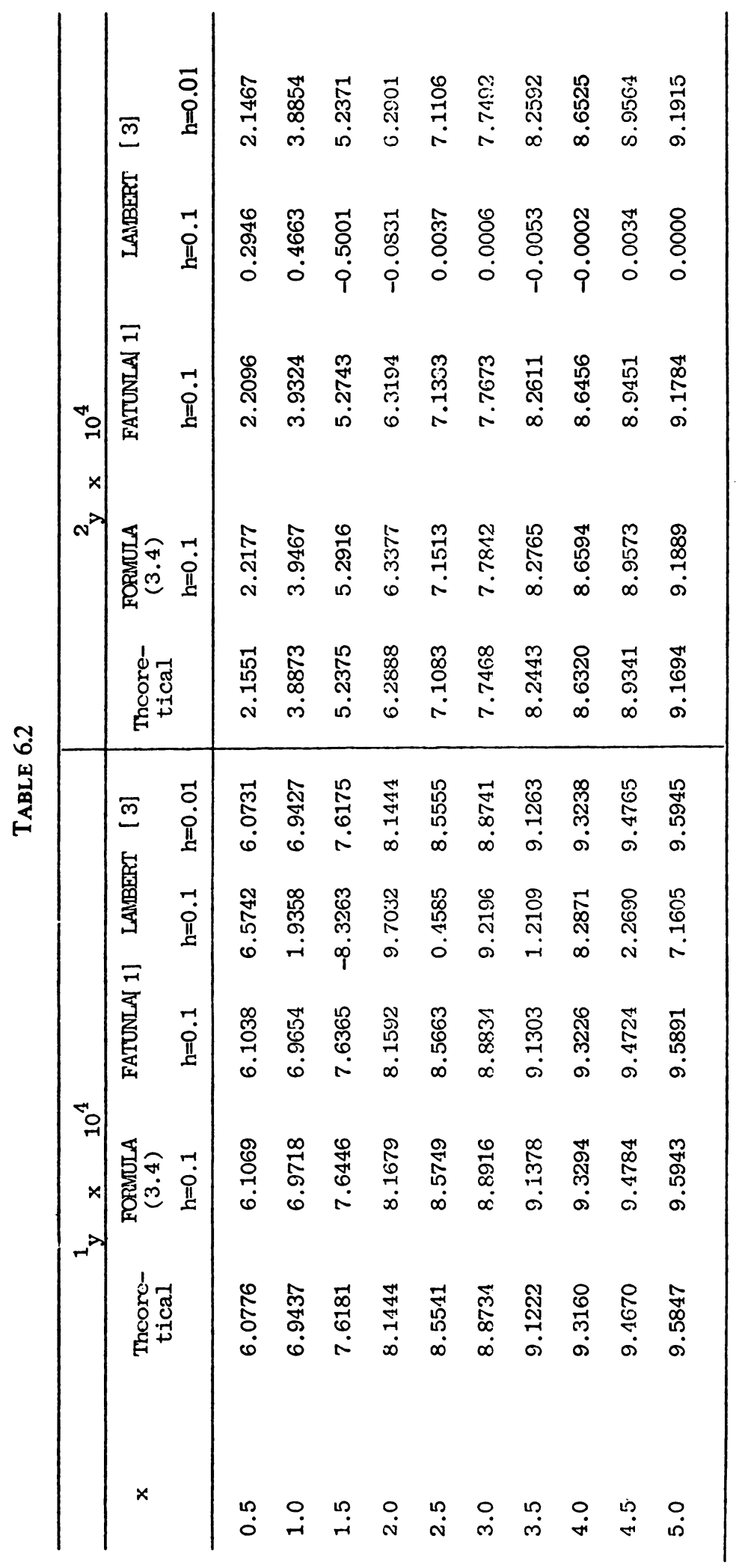


The numerical solution to this problem was effected in the interval $0 \leqslant x \leqslant 100$ with a uniform mesh size $h=0.1$. The stiffness parameters are given by:

$$
\Lambda_{1}=\left(\begin{array}{cc}
-988.63647 & 0 \\
0 & -21.110642
\end{array}\right), \quad \Lambda_{2}=\left(\begin{array}{cc}
1950.56669 & 0 \\
0 & 1011.13956
\end{array}\right)
$$

at $x=0$ and by

$$
\Lambda_{1}=\left(\begin{array}{cc}
-1.31565802 & 0 \\
0 & -1.309375
\end{array}\right), \quad \Lambda_{2}=\left(\begin{array}{cc}
7.71526875 & 0 \\
0 & 7.649220
\end{array}\right)
$$

at $x=100$.

The theoretical solution being unknown was taken as the numerical solution obtained with the fourth order Runge Kutta scheme with mesh size $h=5 \times 10^{-4}$. Lambert used this theoretical solution to start his integration formula at $x=1.0$. The author has his reservation as regards the accuracy of the theoretical solution at $x=100$ because of the tendency for the propagation of roundoff errors which could arise from using such a small mesh size.

The details of the numerical results are given below in Tables $6.3 \mathrm{a}$ and $6.3 \mathrm{~b}$.

TABLE 6.3a

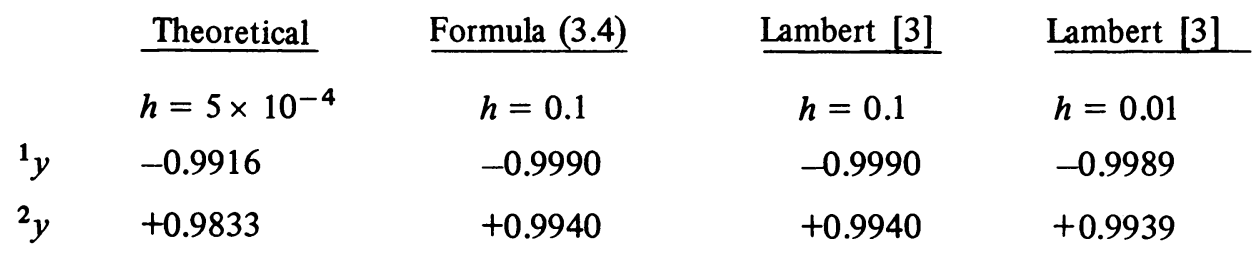

Concluding Remarks. The author is of the opinion that the capability of the new scheme in handling both linear and nonlinear stiff systems with a fairly large mesh size makes it quite competitive with the other existing integration procedure. In cases when $f(x, y)$ is linear, the higher order derivatives are readily obtained by the relationship

$$
{ }^{i} f^{(k)}=\sum_{j=1}^{m} a_{i j}{ }^{j} f^{(k-1)}, \quad i=1, \ldots, m .
$$

Equations (2.13) and (2.14) are solved once for linear systems as the stiffness parameters have constant values.

If, however, $\mathrm{f}(x, \mathrm{y})$ is nonlinear, the higher order derivatives are also readily obtained in terms of the lower derivatives.

The mere fact that no $A$-stable linear multistep method can have order exceeding two is consoling enough in the task of computing ${ }^{i} f^{(j)}, j=1,2,3$.

Although the stability considerations in Section 4 is only confined to diagonal systems, Problem 2 indicates that the new algorithm can effectively cope with nondiagonal systems. 
的总实

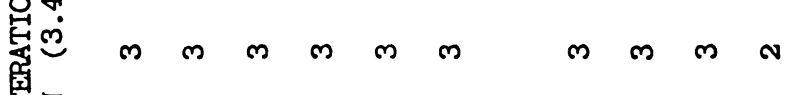
家它

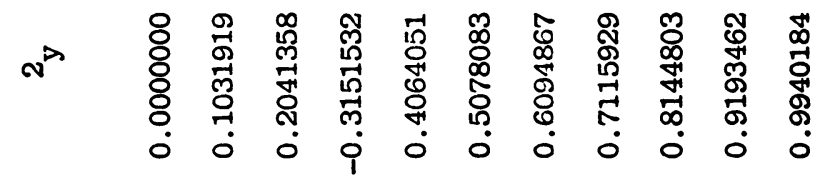

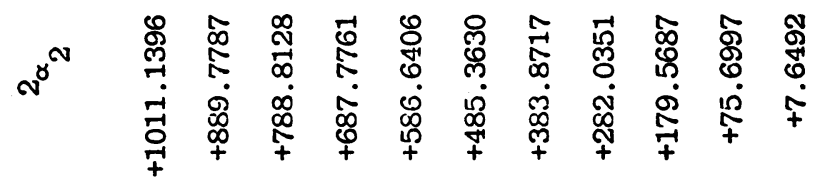

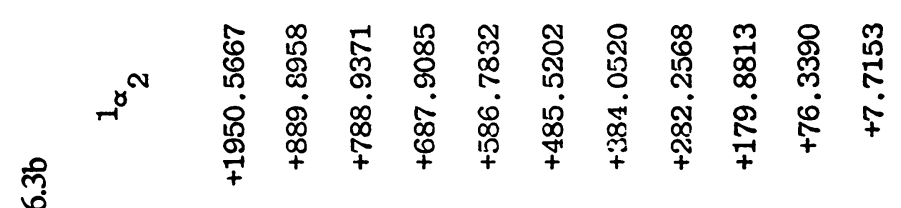

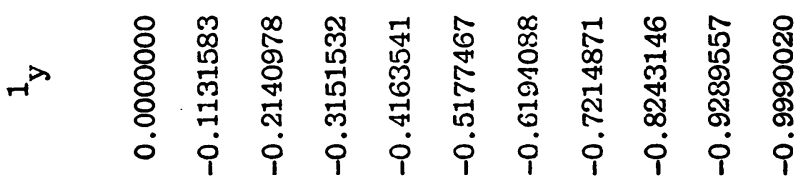

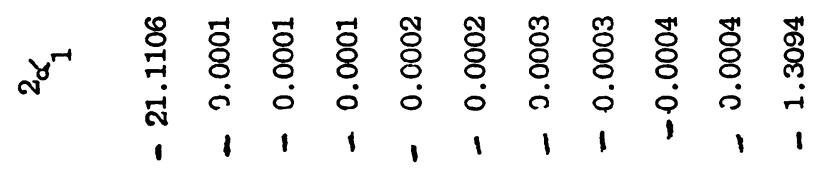

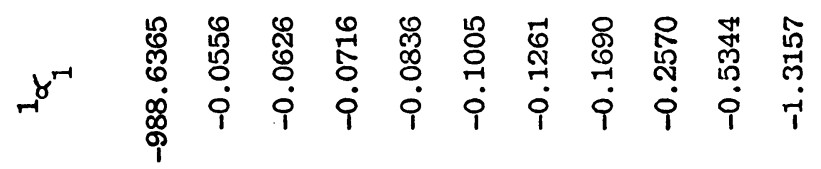

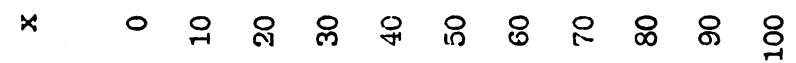


Department of Mathematics

University of Benin

Benin-City, Nigeria

1. S. O. FATUNLA, "A new semi-implicit integration algorithm to cope with stiff systems of ordinary differential equations," Manuscript, 1976.

2. K. M. BROWN, “A quadratically convergent Newton-like method based upon Gaussian elimination,” Math. Comp., v. 20, 1966, pp. 11-20.

3. J. D. LAMBERT, "Nonlinear methods for stiff systems of ordinary differential equations," Conference on the Numerical Solution of Differential Equations (Dundee, 1973), Lecture Notes in Math., vol. 363, Springer-Verlag, Berlin and New York, 1974, pp. 75-88.

4. L. LAPIDUS \& J. H. SEINFELD, Numerical Solution of Ordinary Differential Equations, Academic Press, New York, 1971.

5. M. E. FOWLER \& R. M. WARTEN, "A numerical integration technique for ordinary differential equations with widely separated eigenvalues," IBM J. Res. Develop., v. 11, 1967, pp. $537-543$.

6. W. LINIGER \& R. WILLOUGHBY, "Efficient integration method for stiff systems of ordinary differential equations," SIAM J. Numer. Anal., v. 7, 1970, no. 1, pp. 47-66. 